\title{
EAl Endorsed Transactions

\section{Big Data Management of Hospital Data using Deep Learning and Block-chain Technology: A Systematic Review}

\author{
Nawaz Ejaz ${ }^{1}$, Raza Ramzan ${ }^{1}$, Tooba Maryam $^{1, *}$, Shazia Saqib $^{1}$ \\ ${ }^{1}$ Department of Computer Science, Lahore Garrison University, Lahore, Pakistan
}

\section{Abstract}

The main recompenses of remote and healthcare are sensor-based medical information gathering and remote access to medical data for real-time advice. The large volume of data coming from sensors requires to be handled by implementing deep learning and machine learning algorithms to improve an intelligent knowledge base for providing suitable solutions as and when needed. Electronic medical records (EMR) are mostly stored in a client-server database and are supported by enabling technologies like Internet of Things (IoT), Sensors, cloud, big data, Deep Learning, etc. It is accessed by several users involved like doctors, hospitals, labs, insurance providers, patients, etc. Therefore, data security from illegal access is crucial especially to manage the integrity of data. In this paper, we describe all the basic concepts involved in management and security of such data and proposed a novel system to securely manage the hospital's big data using Deep Learning and Block-Chain technology.

Keywords: Electronic medical records, big data, Security, Block-chain, Deep learning.

Received on 23 December 2020, accepted on 16 March 2021, published on 23 March 2021

Copyright (C) 2021 Nawaz Ejaz et al., licensed to EAI. This is an open access article distributed under the terms of the Creative Commons Attribution license, which permits unlimited use, distribution and reproduction in any medium so long as the original work is properly cited.

doi: 10.4108/eai.23-3-2021.169072

\section{Introduction}

Remote and smart medical care [1] for the aging people and patients monitoring are becoming common among scholars because of its adaption in current socio-economic development where the life of average humans increases leads a life without age-related illnesses also personal care. Electronic Medical Records (EMR) [2] have usually remained stored into scattered records which are commonly tracked on the client-server architecture. To control or accomplish authorization of end-users to produce, access, bring up-to-date, or remove medical data there is server control called administration. Medical information detected through devices is disposed to safety attacks plus weaknesses [3]. In the detecting unit, the data is acquired by numerous small devices. These sensors are vulnerable to harm by fall while physical control leads toward data loss, likewise, sensors might be accommodating via enemy for getting or damaging information, might be exchanged through illegal ones, etc. The record of the devices within communication unit transfer over various communication channels like small-range communication channels for example Wi-Fi, Zigbee, Bluetooth has the changing channel excellence, safety methods, etc. In the loading plus processing unit, the acquired information is kept within the cloud server for future use, handling, information constructing, response or guidance [4], etc. In first stage of this procedure includes detecting of information, the communication of information also storing the information in the cloud. The second stage includes getting information from the cloud, examine or transform information, delete or update information on the cloud via various stakeholders within medical care (Fig.1). Today, for medical care information, integrity plus confidentiality are two main things to confirm hence persons don't have fear about sharing their personal information over illegal access. In medicine, data reliability is important because if data is incorrect then the advice generation is inaccurate. 


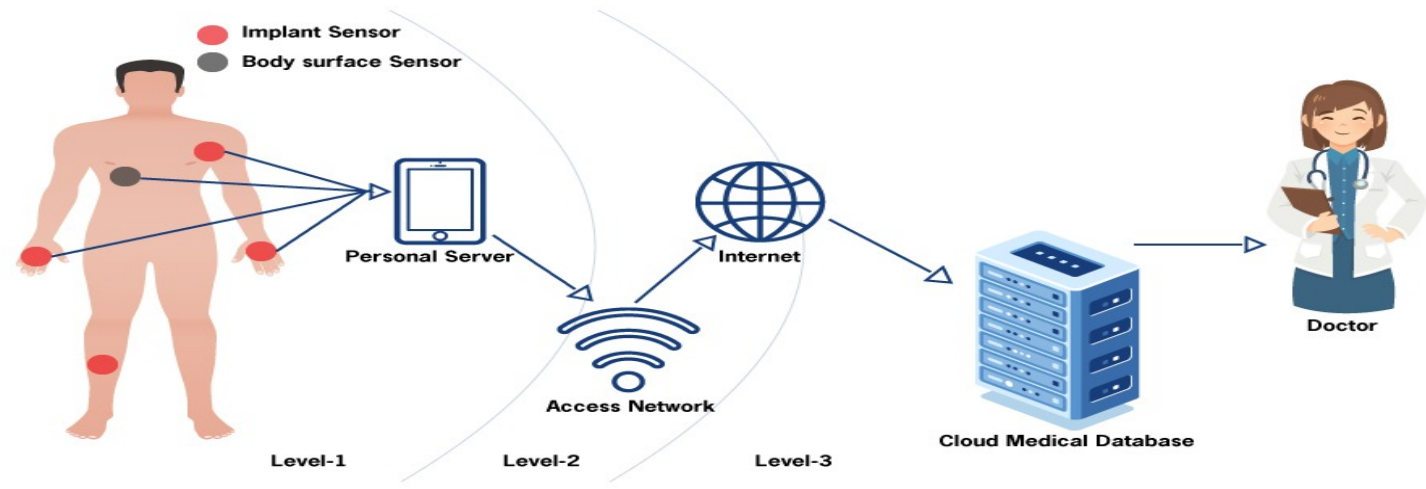

Figure 1. The customary 3-level structural design of wireless body area network (WBAN) established healthcare.

Basic safety methods also value of IoT and cloud-supported medical care structure benefits to avoid other calculations difficulty causing in additional resource consumption because of execution of encryption algorithms independently. Because multiple parties are elaborate in accessing medical information if the technique of information encryption at the source side and then decryption at the corresponding destination then it takes a lot of time which is not tolerable in applications like medical care. Also, sensors in IoT-supported remote medical care organizations are diversified having changing resource levels. Therefore, individual encoding and decoding procedures like Advanced Encryption Standard (AES), Data Encryption Standard (DES), and Rivets-Shamir-Adelman (RSA) may not be useful in all stages of remote medical care architecture. Therefore, in confidentiality, there is a deployment problem but confidentiality might be confirmed via executing verification plus reliability. There is a distributed network in block-chain [5], where investors (surgeons, hospitals, inquiry laboratories, and insurance providers) are linked with each other's is known as blockchain nodes. Medical Devices gather information and refer it to the Personal Digital Assistant (PDA) which forwards information to the block-chain link over admittance facts. The information transferred to the block-chain channel in a single term stays fair title to a block. Hash of the past information is assured by latest information, therefore, block-chain system can validate the latest block of information, when the authenticated hash of the information is saved in block-chain nodules plus the medical data is stockpiled within the cloud record in encoded layout. Large medical information [6] saved within cloud Records needs more investigation using Machine learning methods for data removal. Deep Learning methods are today commonly used inside medical care; various common applications include initial illness discovery, DNA (Deoxyribonucleic Acid) analysis, estimate of the latest medicines efficiency, modified cures, etc. The large challenge of utilizing deep learning methods in medical material is requisite for a massive quantity of categorized information. EMR can hold diverse un-labeled information, e.g. X-ray images without any medicinal health situations similar to fibrosis or cancer. In this type of situation, unsupervised learning methods may have used for the classification of information using data mining. Supervised learning can be used for categorized information. And semi-supervised learning is useful for both categorized and uncategorized information. Convolution Neural Network $(\mathrm{CNN})$ is an extremely obstructed Deep learning method amid extra like Deep Neural Network (DNN), Deep Autoencoder, Deep Belief Network (DBN), Recurrent Neural Network (RNN) by way of medical records is predominance picture-centered at the moment. DNNs in actualtime requests such as medical care have effectively been applied with the parallel provision of Graphical Processing Units (GPUs) [7].

Section II gives an overview of related work done in this field and presents the novelty of our work. Section III describes the base technologies that are used for the management and security of hospital's Big data. Section IV presents the hospital system's security model along with possible attacks on such systems. A detailed proposed solution architecture, for secure management of Hospital's Big data, with the description of all the components is presented in section V. Section VI discusses the open research challenges in this research area and Section VI concludes the paper and gives future perspectives.

\section{Related Work}

In this section, we explained the work related to big medical data and the problems towards its smart processing via Deep learning methods. Moreover, the safety of medical records in terms of authentication, integrity, and privacy is most important. Therefore, efficient safety methods are focused certainly to confirm the safety of medical records and fixing the issues and challenges associated with medical records. In [7], journalists have explained a rough analysis of deep learning methods and execution problems in processing big medical records in terms of past scope, advantages, and 
disadvantages. The use of deep learning is also focused on both image-based figures and sensors-based medical data. In the medical field, EMR consists of health records of the patient like medical test reports, treatment and follow-up, medicines record, EEG, ECG or EMG signals, sensors data by using universal sensors like heart-rate, blood pressure, fever, pulse-rate, etc. Medical information in the medical field is not labeled and complete every time. This type of information might be inaccurate.

Table 1. Application of many deep learning techniques in medical informatics.

\begin{tabular}{|l|c|c|c|c|c|}
\hline \multicolumn{1}{|c|}{ Authors, year } & \multicolumn{4}{|c|}{ Security requirements considered } & Implementation \\
\cline { 2 - 5 } & Authentication & Confidentiality & Integrity & Access control & \\
\hline Zhang et al. [26], 2018 & $\checkmark$ & $\checkmark$ & $\checkmark$ & $\times$ & $\checkmark$ \\
\hline Azaria et al. [27], 2016 & $\checkmark$ & $\checkmark$ & $\times$ & $\times$ & $\times$ \\
\hline Peterson et al. [28], 2016 & $\checkmark$ & $\checkmark$ & $\times$ & $\times$ & $\times$ \\
\hline Patel et al. [29], 2018 & $\checkmark$ & $\checkmark$ & $\times$ & $\times$ & $\times$ \\
\hline Xia et al. [9], 2017 & $\checkmark$ & $\checkmark$ & $\times$ & $\checkmark$ & $\checkmark$ \\
\hline
\end{tabular}

Also, the data is detecting through various devices, so that the format and size of the data are not the same. The sequence of data acquired is also a big challenge in the handling of that datasets. Open defies in the use of deep learning methods like CNN, DNN, etc. in huge medical data handling deceits in the fauna of this method aforementioned. Lack of complete and accurate exercise data will cause the pitiable exercise of the DNN model. Moreover, incomplete training data may cause fewer errors but lead to a high rate of errors while testing with other datasets. Scholars often use deep learning methods like the CNN model. Accurate processing, normalization, and filtering of the training dataset are most significant as noise may cause incorrect classification of data in machine learning methods like logistic regression, etc. Instead of this, if suitable clarification of hyper-parameters can be made up, the DNN configurations, several filters within CNN can be organized and pre-explained. In this perspective, block-chain-centered health information distribution between un-trusted parties confirming authentication, access control, and confidentiality has been offered [9]. To overcome illegitimate modification by the intruder, transactions request between authentic parties to get information from the cloud are protected with encryption keys. Threat models have been established to detecting safety attacks and medical data threats as well as health reports. When someone requests to access data, the signature-based verification is completed before retrieval of data. This data is encoded and directed toward the requestor. The reason for the request for data right to use is also measured. When the number of requestor's increases performance assessment in terms of latency is done based on the actual test situations. In [10] writers have offered a difficult and reasonable analysis of associated work on block-chain and its applications in medical care. This method scientifically find features of block-chain technique that's make it appropriate to confirm safe and reliable communications between numerous investors on the public health data of the patients. A huge amount of publications has been evaluated and analyzed based on related constraints like smart contracts, block-chain platform used, variety of block-chain system, consensus algorithm implemented, etc. This exertion includes hard research of block-chain papers in medical care throughout the period in the middle of 2008-2019 to find that a decent value effort of investigation has been issued merely 2015 ahead and executed work have been issued in 2017, in 2018 the notice in block-chain centered safe medical maintenance is increased. However, block-chain is defined as efficient and capable method to grip public health data between various involved parties confirming confidentiality, authentication, access control, integrity, etc., in block-chain, there are numerous trials and limitations in the framework of actual medical care situation. In [11], writers identifies various challenges e.g., storage, other expenses in the relation to the statement, postponement in the execution of appeals to right to use data, scalability problems. Also, the assessment of the working of offered block-chain-centered system has been ended. The analysis of the use of deep learning methods in medical care has been explained in [12]. They have characterized deep learning methods realistic to particular biological indications like EEG, ECG, and EMG. A comprehensive design on techniques of deep learning in cooperation scientifically and design astute will fascinate potential or current scholars in associated regions. Writers in [13] offered an exclusive safe smart medical care method using smart agreements by applying a remote block-chain established on Ethereum procedure. Ethereum built remote block-chain confirms that simply real consumers can right of entering patients' medical information. In this research, only the measures like data handling in remote devices by using smart contracts, data detection from devices, alert generation and transfer to care-givers, etc. are kept in the block-chain record. Genuine confidential data correlated to health data is stored in EMR and plotting is retained between block-chain and EMR record to right to use or recover information. While emerging and applying for this work few restrictions have been classified e.g. latency, structured key managing, etc. 
Table 2. Usage of block-chain technology in the medical data distribution.

\begin{tabular}{|l|l|l|l|}
\hline \multicolumn{1}{|c|}{ Authors, year } & \multicolumn{1}{|c|}{ Application } & \multicolumn{1}{c|}{ Input data Deep } & Deep learning method \\
\hline Fakoor et al. [16], 2013 & $\begin{array}{l}\text { Cancer diagnosis and } \\
\text { classification }\end{array}$ & Gene expression & Deep Auto Encoder \\
\hline Rongjian et al. [17], 2014 & Brain Disease Diagnosis & $\begin{array}{l}\text { Alzheimer's disease } \\
\text { neuroimaging initiative(ADNI) } \\
\text { dataset }\end{array}$ & CNN \\
\hline Yaniv et al. [18], 2015 & Chest pathology detection & X-ray images & CNN \\
\hline Ramsundar et al. [19], 2015 & Drug Discovery & Molecular Compound & DNN \\
\hline Sun et al. [20], 2016 & Lung cancer diagnosis & $\begin{array}{l}\text { Lung Image Database } \\
\text { Consortium (LIDC) dataset }\end{array}$ & CNN \\
\hline Charissa et al. [21], 2016 & Human activity recognition & Raw sensor data & CNN \\
\hline Ahmed et al. [22], 2016 & Breast cancer classification & $\begin{array}{l}\text { Wisconsin breast cancer dataset } \\
\text { (WBCD) }\end{array}$ & DBN \\
\hline Mohsen et al. [23], 2017 & Brain tumor classification & $\begin{array}{l}\text { Magnetic resonance imaging } \\
\text { (MRI) images }\end{array}$ & DNN \\
\hline Esteva et al. [24], 2017 & Skin cancer classification & Clinical imaging & CNN \\
\hline Amin et al. [25], 2018 & Brain tumor detection & $\begin{array}{l}\text { Magnetic resonance imaging } \\
\text { (MRI) images }\end{array}$ & DNN \\
\hline
\end{tabular}

In an IoT-supported system, the number of devices is high and will increases quickly above phase hence key peer group and sharing is a minor problem. Such systems provide help in emergency medical situations by decreasing invisibility in block-chain record handling is similarly a genuine task. A modern investigation [14] has offered CNN-based methods for the prediction of chronic disease risks. Journalists collect the real-life hospital records for regional chronicle diseases from the hospitals of China, as medical records are generally uncharacterized and consist of misplaced information, they use an underlying aspects model to regenerate the misplaced data. They have applied their applications and associated them with other diseases predictions algorithms.

The new outcomes confirm the exactness of $94.8 \%$ and a merging rapidity that is relatively improved. In [15] writers show that few machine learning systems are susceptible to a safety attack acknowledged as exterminating attacks. In these types of attacks, the attackers increase the training datasets using spiteful data which reasons incorrect outcomes than predicted. This is very dangerous while detecting a patient's disease. In conclusion, journalists offered avoidance methods for this type of threat. In Table1, we have proposed some work issued in the previous years implementing Deep Learning related procedures in medical care uses. Table.2 demonstrates the mechanism for the distribution of medical information utilizing a block-chain.

In literature, Dee Learning is being used by researchers to manage big data efficiently in real-time. Block-chain is also being used to maintain the integrity of the data. We used both these techniques together and proposed a system that securely manages the big data of hospitals.

\section{Preliminaries}

In this segment, discussion about innovative technologies like the Internet of Things (IoT), big data, different Deep Learning procedures, also block-chain have done briefly.

\subsection{Internet of Things (loT)}

The vision driving IoT is a system interconnected with each another for data transmission over a network requires human to human or human to computer interaction [30]. Drastically IoT is growing in healthcare. It is beneficial for both physicians and patients. For example, with the use of IoT connectivity patients can take precautions from the doctor without visiting and provides real-time data to the doctor for better treatment, they don't need to pay a visit. By availing sources like sensors and network data can be retrieved and sent over devices remotely. Remote healthcare is needing nowadays so IoT is core technology as a solution. The IoT can be wearable or implantable powered by lightweight batteries. It forwards the data obtained through sensors transmitted over the network to medical facilitation like hospitals or clinics etc. Having this data that is increasing speedily which can be determined as "big data" needed to be protected, but at the same time can be available to all participants. 


\subsection{Big Data}

Big data is incredibly huge data sets, regardless of organized, unstructured, and semi-structured arrangement. Arranged data contains indexes or spreadsheets in an arranged manner. Unstructured data consist of audio, video, and images, generally they are difficult to analyze if present in abundance. Without standards to be followed by semi-structured data like XML. All these data play a vital role in growing technological applications like the medical suggestion, disease predictor, Drug records, medicine recommender, etc. traditional software are unable to manage these data in bulk, so enormous data needed to be secured and available by using of cloud platforms that save cost for storing and sharing.

\subsection{Deep Learning}

Artificial neural networks are stimulated by humans' brain which meets machine learning for solving complex problems. So, machine learning has a subset called deep learning (DL). DL method is used to draw out features from bulk data, collecting useful information from big data using DL algorithms is useful in many aspects. Since detecting a feature is time-consuming and costs a lot. DL methods do not require labeled data for learning objectives. In case we may have both types of data labeled and un-labeled in healthcare like x-rays images regardless of a medical condition, huge data that is not labeled, etc.

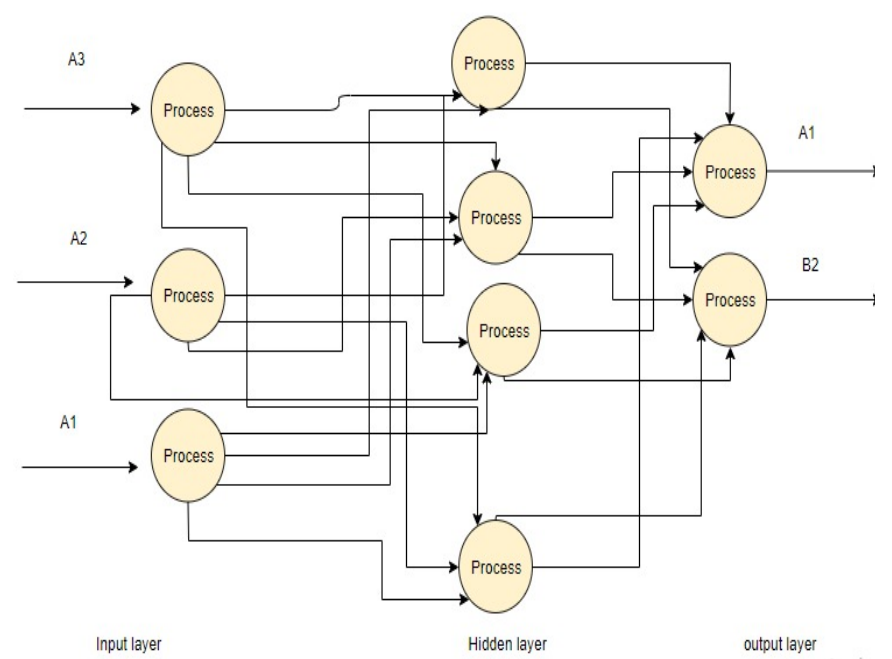

Figures 2. The Structure of an Artificial Neural Network (ANN).

There are many Deep learning techniques. In this portion, we discussed some of the widespread among them.

(1) Artificial Neural network (ANN) is a DL technique that contains several organized layers which have perceptron, the neurons. Multiple layers are starting from the input layer then the hidden layer, actually this works as the training layer and output layer. If hidden layers are increased vastly then it may not assure improved results. Overfitting also takes place if many layers are added at the same time as a result many distortions forwarding too much interference in data being captured. Fig.2 Convolutional neural network (CNN) until now highlighted beneficiary technique in healthcare. A static extent flight path is using as an input. It can be applied to medical data like in image processing for the detection of the tumor. Perceptrons interconnected with each other and assigned a weight to them that can be adjusted after each iteration. As the propagating waves are transmitted in one direction i.e. starting from input meanwhile ending on the output layer is known as a feed-forward network.

\subsection{Popular Deep Learners}

Several deep learning methods are open; we have to select intelligently the finest method for precise delinquents. Table. 3 demonstrates standard approaches which have been used in health informatics.

\subsubsection{Convolutional Neural Network}

Convolutional neural network $(\mathrm{CNN})$ is enthused by the human cortex and is one of the supreme prevalent deep learning methods among others. It is considered as a feedforward network containing multiple layers implies in one way from input to output. When data is passed through the process layer, useful features are extracted from input data, and the result is shown in output layers. In healthcare, it is used for disease detection from sample tissues. It promotes read structures usually difficult to understand by human medical experts.

\subsubsection{Recurrent Neural Network}

In healthcare, another major technique is the Recurrent neural network (RNN), for data to be examined additionally (RNN) is used as it delivers streaming. Perceptrons are interlinked which behaves as storage for continuous inputs. In healthcare, it can be applied in several analyses like anamnesis. Like many infect ants have the same disease with different symptoms RNN can find similarities then it would be better for physicians to cure.

It is a method of unsupervised learning in which linking between numerous multilayers is pointless and it comprises numerous unseen layers. Like we have two layers naming even and odd layers oppose each other adjacent, it can be preserved as a two-part chart. No presence of interlayer connection is available in (DBM), since the neighboring layers are associated but essentially it is slow.

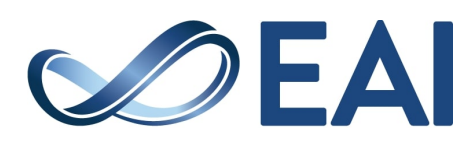




\subsubsection{Deep Autoencoders}

Past studies illustrate that there is no generic feature that works precisely on different data sets. The data-driven technique is more accurate for feature withdrawal. So Auto encoder's neural networks are presented. In this method, an identical number of input and output is used using an unsupervised technique. Hidden layers are less in number than input and output layers. For extrication of significant characteristics, it conceals the statistics in lower-dimensional space.

\subsubsection{Deep Boltzmann Machine}

It is a method of unsupervised learning in which linking between numerous multi-layers is pointless and it comprises numerous unseen layers. Like we have two layers naming even and odd layers oppose each other adjacent, it can be preserved as a two-part chart. No presence of interlayer connection is available in (DBM), since the neighboring layers are associated but essentially it is slow.

Table 3. instant of prevalent deep learning structure.

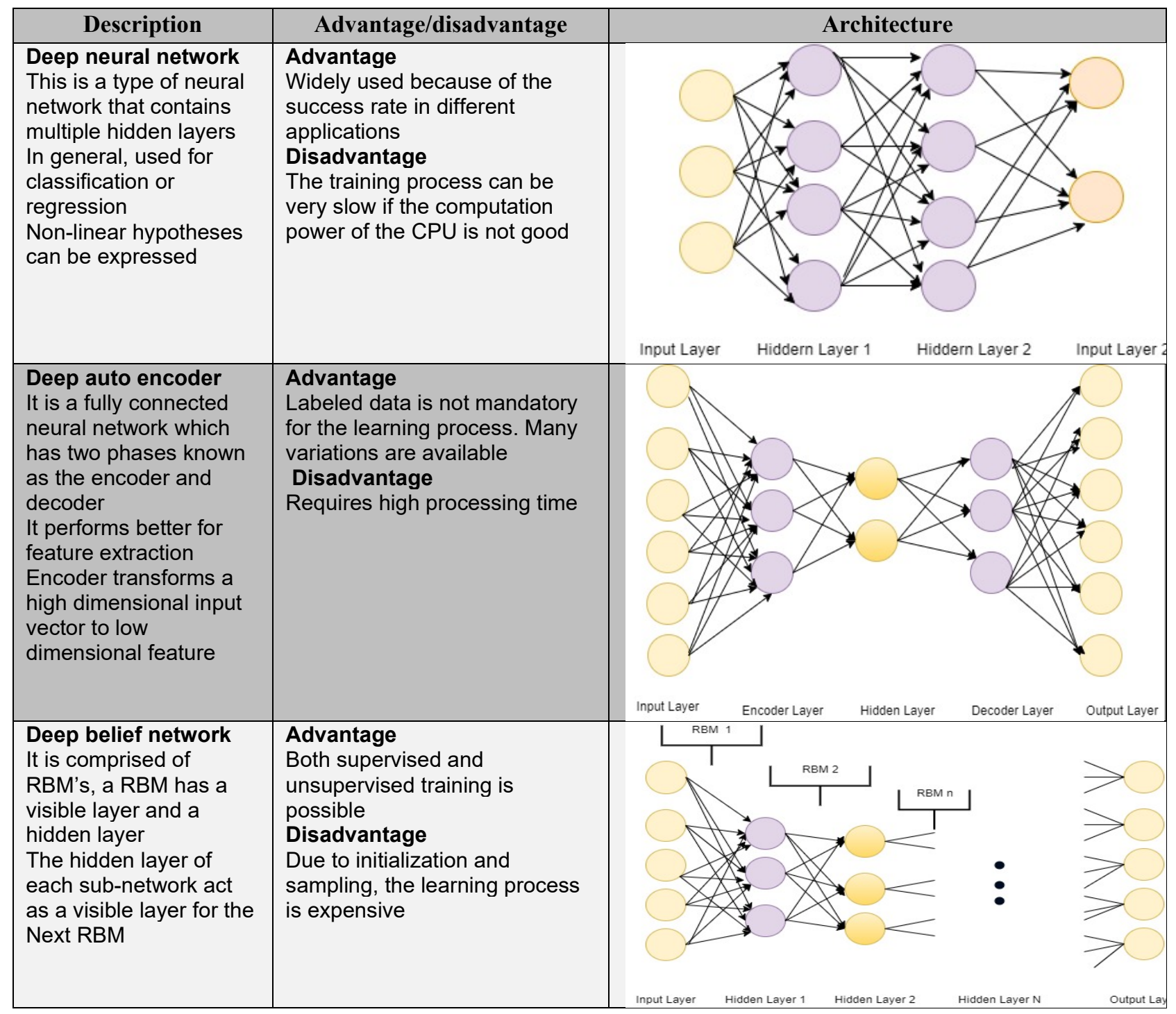




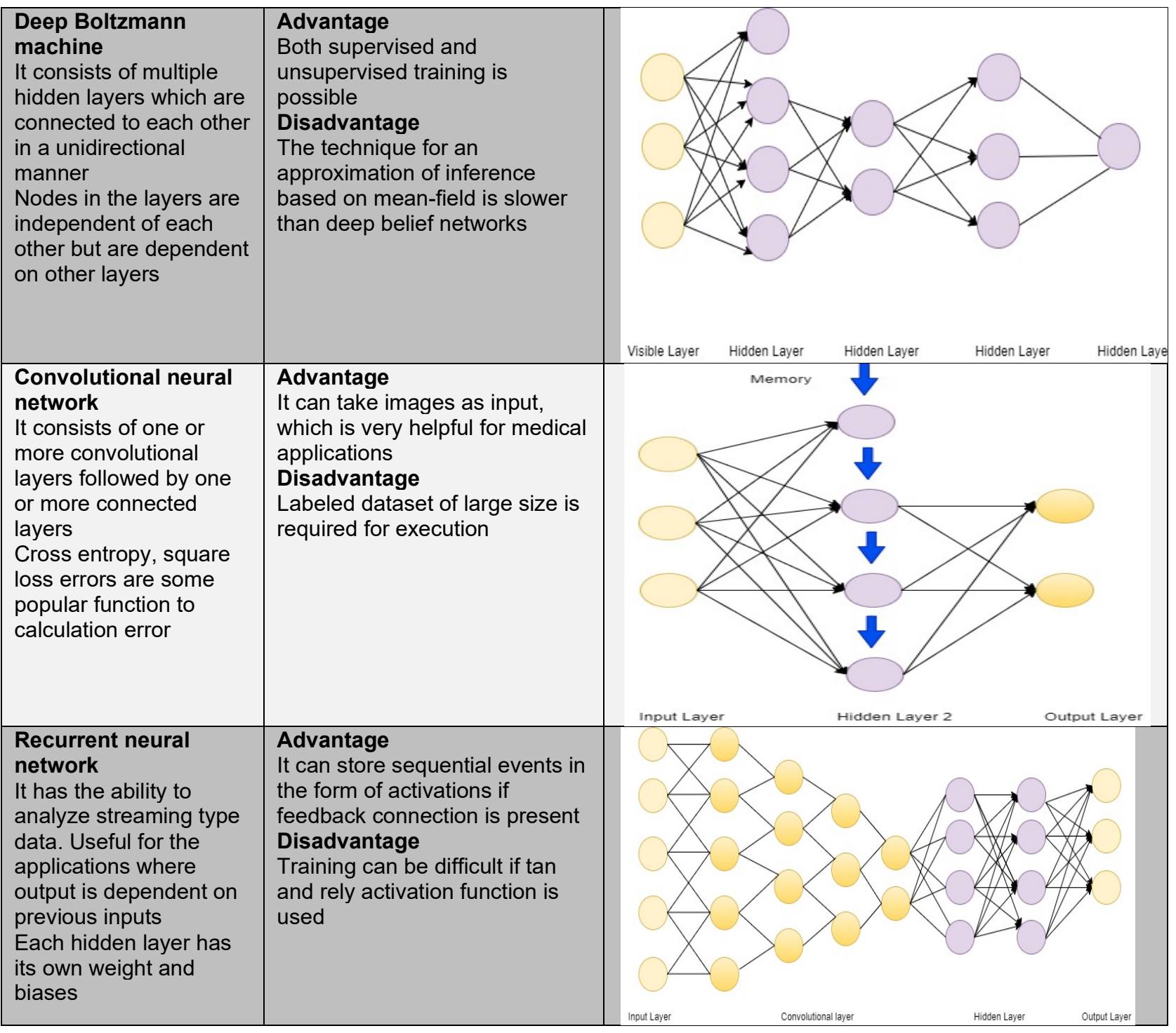

\subsubsection{Restricted Boltzmann Machine}

The Boltzmann machine has many variants one of the most popular is the restricted Boltzmann machine (RBM). It comprises of random probability distribution but may not be resultant precisely. Gibbs sampling is some procedure in the learning process. In RBM nodes are undirected, propagation of values in every direction. Contrastive Divergence (CD) algorithm is utmost shared way to make RBM learn. The stages of $C D$ algorithms are considered as constructive and adverse. The set of training is simulated by change in connection formation and in negative stage data used to be reconstructed based on present network configuration.

\subsubsection{Deep Belief Network}

The frame work of Deep Boltzmann machine can be taken as Deep Belief Network (DBN). It also consists of hidden layers in which every second network is allied to observable layer of RBM. It has both directed and undirected connection like for top two layers connection are undirected likewise directed for lower layers. Greedy learning method used layer by layer to start DBN, and progressively adjustments are thru to accomplish goals. Some prevalent Deep learning methods are summed up in Table 3. Table 4 demonstrate current standard software packages where a neural network can be implemented.

\subsection{Applications and Challenges of Deep Learning}

AI (ML) has numerous effective applications in the wellbeing zone of facts but Deep Learning (DL) procedures and their selection are later Is slightly moderate. Nevertheless, DL has rapid success and can guarantee results irrespective of the Challenges ahead. We are able to classify clinical DL uses into three groups. 
- Prescient Healthcare, e.g. Efficiency of predicted treatment for different diseases.

- Clinical Decision Support, e.g. different maladies can be identified and analyzed utilizing physiological data of the patient.

- Customized Therapies, e.g. Specific medications should be formulated according to the needs of the individual sufferers.

\subsubsection{Prescient Healthcare}

This type of use is intended to identify diseases in the early stages with the objective and that surgery can be started before the sufferers enters a basic condition. By and large, identification of Alzheimer is troublesome in its beginning period. Different zones of prescient medicinal services incorporate anticipating the adequacy of medicines. For model radiographs or automated center tomography (CAT) clearing, significant examination (DL) can be utilized to perceive peculiarities that are hard to distinguish by the regular eye.

Table 4. Software Apparatuses for the execution of neural networks.

\begin{tabular}{|c|c|c|c|c|c|c|c|}
\hline \multirow[t]{2}{*}{ Software } & \multirow[t]{2}{*}{ Developer } & \multirow[t]{2}{*}{ Platform } & \multicolumn{4}{|c|}{ Supported method } & \multirow{2}{*}{$\begin{array}{c}\text { Cloud } \\
\text { support }\end{array}$} \\
\hline & & & CNN & RNN & DBN & RBM & \\
\hline $\begin{array}{l}\text { Neural Designer } \\
\text { [31] }\end{array}$ & Microsoft & $\begin{array}{l}\text { Microsoft } \\
\text { Windows, Linux }\end{array}$ & $\checkmark$ & $\checkmark$ & $\checkmark$ & $x$ & $\checkmark$ \\
\hline Keras [32] & François Chollet & $\begin{array}{l}\text { Microsoft } \\
\text { Windows, Linux }\end{array}$ & $\checkmark$ & $\checkmark$ & $\checkmark$ & $x$ & $x$ \\
\hline $\begin{array}{l}\text { Apache SINGA } \\
\text { [33] }\end{array}$ & $\begin{array}{l}\text { Apache Software } \\
\text { Foundation }\end{array}$ & $\begin{array}{l}\text { Linux, macOS, } \\
\text { Window }\end{array}$ & $\checkmark$ & $\checkmark$ & $x$ & $\checkmark$ & $x$ \\
\hline $\begin{array}{l}\text { Deeplearning } 4 \mathrm{j} \\
\text { [34] }\end{array}$ & $\begin{array}{l}\text { Adam Gibson, Josh } \\
\text { Patterson }\end{array}$ & $\begin{array}{l}\text { Linux, macOS, } \\
\text { Windows, Android }\end{array}$ & $\checkmark$ & $\checkmark$ & $\checkmark$ & $\checkmark$ & $x$ \\
\hline $\begin{array}{l}\text { Microsoft } \\
\text { Cognitive Toolkit } \\
{[35]}\end{array}$ & Microsoft Research & Windows, Linux & $\checkmark$ & $\checkmark$ & $x$ & $x$ & $x$ \\
\hline $\begin{array}{l}\text { Apache MXNet } \\
\text { [36] }\end{array}$ & $\begin{array}{l}\text { Apache Software } \\
\text { Foundation }\end{array}$ & $\begin{array}{l}\text { Windows, macOS, } \\
\text { Linux }\end{array}$ & $\checkmark$ & $\checkmark$ & $x$ & $\checkmark$ & $\checkmark$ \\
\hline OpenNN [37] & Artelnics & $\begin{array}{l}\text { Microsoft } \\
\text { Windows, Linux }\end{array}$ & $\checkmark$ & $\checkmark$ & $x$ & $x$ & $x$ \\
\hline PyTorch [38] & Adam Paszke & $\begin{array}{l}\text { Linux, macOS, } \\
\text { Windows }\end{array}$ & $\checkmark$ & $\checkmark$ & $\checkmark$ & $\checkmark$ & $x$ \\
\hline TensorFlow [39] & Google Brain & $\begin{array}{l}\text { Linux, macOS, } \\
\text { Windows, Android }\end{array}$ & $\checkmark$ & $\checkmark$ & $\checkmark$ & $\checkmark$ & $x$ \\
\hline Theano [40] & $\begin{array}{l}\text { Montreal Institute for } \\
\text { Learning Algorithms } \\
\text { (MILA) }\end{array}$ & $\begin{array}{l}\text { Linux, macOS, } \\
\text { Windows }\end{array}$ & $\checkmark$ & $\checkmark$ & $x$ & $\checkmark$ & $x$ \\
\hline
\end{tabular}

DL can be a lot successful in oddity identification since it can recognize little varieties which can stay undetected by the human in beginning periods. Clinical pictures are anything but difficult to get and it tends to be utilized as preparing information which can tackle the scanty information issue. Conduct information of patients can be likewise utilized for the early identification of disease. Utilizing this diverse clinical information DL can assemble an expectation model. Another significant part of insightful human administrations can be a desire for the adequacy of new drugs. As of not long ago, results are not empowering, however, new upgrades can be made.

\subsubsection{Clinical Decision Support}

A major application of Deep Learning in health information technology is clinical Decision support which is nowadays very trendy. Profound Learning procedures assist the specialists in each phase of a clinical

determination like the discovery of the disease, custom treatment proposal, and post-treatment and so on. On account of malady forecast from picture investigation, DL (Deep Learning) methods can be increasingly exact than people. Biomedicine content examination should be possible through Deep learning. Because of area autonomous nature, any sort of information can be broke down just as connected utilizing DL. Relationship investigation should be possible utilizing an alternate sort of electronic wellbeing history of patients to give a superior conclusion. Likewise, from a solitary informational collection, relationship examination should be possible, for instance, cerebrum locales can be connected from various X-ray pictures. For relationship investigation, $\mathrm{CNN}$ procedures are broadly utilized. CNN 
can make deliberations of the info information even information are gathered from heterogeneous sensors. A clinical specialist will be unable to experience a major clinical data of a patient. Hence, DL can carry out that responsibility and can give clinical choice help.

\subsubsection{Customized Therapies}

Customized medicines are firmly identified with clinical choice help. In light of the expectation, Deep Learning procedures can bolster dynamic and consequently, customized medicines can be given also medications can be planned. Electronic wellbeing records put away at cloud database are for the most part multimodal and unstructured and because of the ongoing headway of advancements, DL can offer a finding dependent on the information. Customized medicines can be available dependent on different information. For instance, biomarkers can be dictated by DNA investigation and the extraction of Genomes. Biomarkers are only a natural state (illness) pointer that can be estimated. Every ailment is created by itself within the humanoid body. Biomarkers can decide on this likelihood of improvement, and this can aid clinical specialists deliver better forecasting and findings. Genomics assists in the identification of a specific gene that is likely to boost an ailment. The adequacy of medication can be dictated by evaluating the qualities distinctions when the medication is put in, called pharmacology. This assists with lessening the dose levels just as the symptoms of the medication. Profound Learning strategies perform very well in malignancy order from quality articulation information.

For instance, to anticipate grafting design highlights Utilizing DL, removal from Ribonucleic Acid (RNA) and Micro Ribonucleic Acid information could be accomplished viably. Deep learning can assist with assessing EMR information thusly and can convey customized drugs.

\subsubsection{Challenges}

DL (Deep learning) is having multiple difficulties in the field of wellness knowledge. According to the nature of meds, there is a prerequisite of security, accessibility, unwavering quality, productivity. For instance, a wellbeing sensor must work consistently with no interference, so crises can be dealt with. Some recent plays show the significance of Filters may be used in $\mathrm{CNN}$ to remove high-level features, be that as it may, the whole learning module won't be explicable. The vast majority of explores use Deep learning procedures without knowing the chance of progress; on the off chance that misclassification issues happen, at that point they don't be able to adjust. We have talked about in the past segments that huge datasets are required for a viable and solid preparing model. These days tremendous human services information is accessible yet sickness explicit information is as yet constrained. Deep Learning is not proper for applications including extraordinary illnesses. Another fundamental issue in the arranging of Deep Neural Network is overfitting while using the little dataset for the arrangement. This happens if the hard and fast number of tests in the preparation set is similar to the proportion of framework in that strategy. The issue of overfitting might be fended off by abuse of regularization methods, for example, dropout during the planning strategy. DNN doesn't authentically reinforce rough data as data; some preprocessing is required thusly, or the data space ought to be changed. For instance, the amount of diverts in CNN, hyperparameters that control the building of a DNN is an outwardly debilitated examination process and exact endorsement is a ton required. It is a troublesome task to locate a perfect course of action of the hyper framework and right Retrieval of unrefined fact, and it could provoke the long-lasting arrangement process. Another significant problem in Deep Learning is multiple DNNs could be tricked effectively if there would a small change in the insertion of information (including indistinct commotion in a picture) then the examples will be misclassified[41] at that point. It tends to be noticed that this problem can influence the vast majority of AI calculations. On the off chance that the estimation of a specific element is set to low or strong, the issue of misstated in calculated relapse will emerge. In selection trees, if a solitary parallel portion is exchanged in the last layer, it will then item off-base outcomes at that stage. Thus, we can say some AI method is defenseless for security assaults likewise, as a basic modification Starts to lead the pack framework to deliver wrong outcomes.

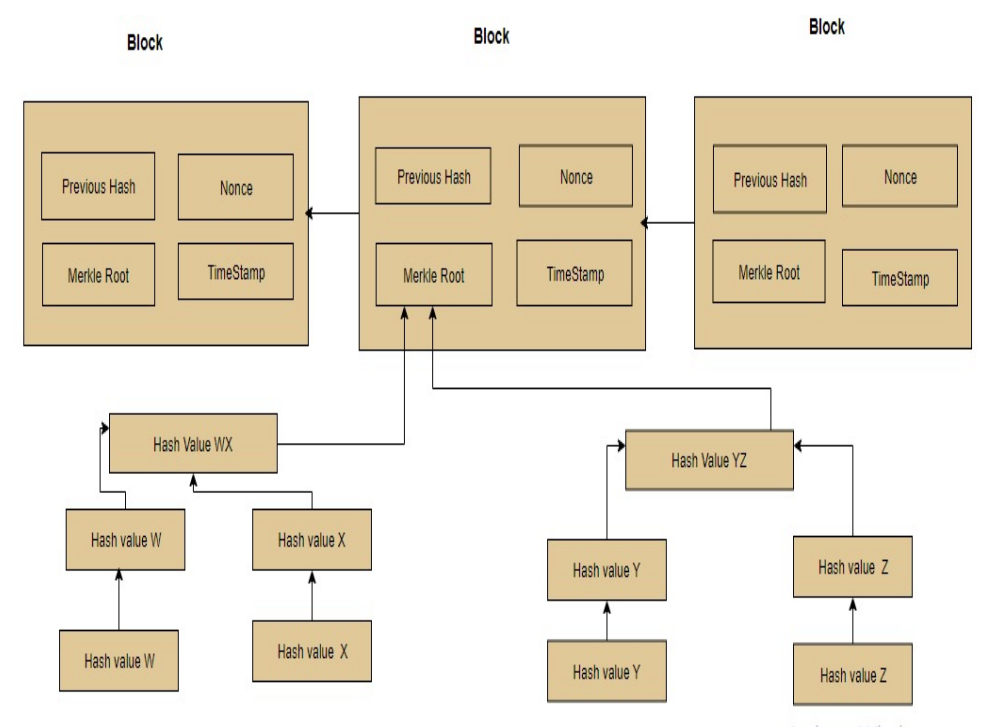

Figure 3. Architecture of a block in block-chain 


\subsection{Block-chain Technology}

A block-chain is a pool of de-centralized node/CPU wherever data can be stockpiled in blocks. It's as well recognized as a de-centralized ledger wherever records chunks are modernized constantly (Fig. 3) Records chunks main contains settlements, pacts, auctions, financial transactions, health records etc. block-chain was presented by Satoshi Nakamoto in year of 2008. Mainly it was established to protect crypto currency (Bitcoin) dealings. Nevertheless now a days these peer-to-peer equipment has been implemented via numerous divisions alike economics, transport, learning, health-care, governance, etc. Cryptographic algorithm mark the method temper resilient; these algorithms mark the method computationally incredible to modify data/transactions stockpiled in the block-chain. Interloper needs to be conciliation $51 \%$ of $\mathrm{CPU} /$ knobs to incredulous mincing control of under attack block net. A chunk comprises a title and a note. Numerous strictures practice the title are as mentioned: (1) Times-tamp which record severe period of formation of a chunk, (2) Preceding chunk mess denotes to muddle of an earlier chunk of sequence, (3) Markel origin which comprises a mess of origin of a shrub that is SHA256 Mess of dealings (4) Struggle mark is unknown however a part of records which is tough to attain fixed via proof of work (PoW) algorithms (5) Nonce is obligatory to attain exertion mark, it correspondingly protect response outbreaks. Every chunk is inter-connected over the mess of the earlier chunk. On the further side, the message comprises the hash of preceding dealings, dealings/message to be directed, and a digital signature of the proprietor. This digital signature is preserved as the resilient possession of deal/message which can be confirmed via the community key of the proprietor. Respectively time a block is permitted and connect to block-chain that turn into unchallengeable which can't be mitigated.

\subsection{Categories of block-chain}

In common, many categories of block-chain can be contingent on fared data, accessibility of the data, and action accomplished by a consumer. We can classify in there

1. Community Block-chain (Permission-less)

2. Consortium (Community Permission-able)

3. Private Block-chain

As of the categories of block-chain, it is vibrant that block-chain which are reachable and observable to the community are community block-chain. Though, the intact data might not be reachable by the community, meanwhile specific portion of the data can be in an encoded setup to keep members secrecy [43]. In the community block-chain, anybody can link the block-chain and doing as a node, or can convert into a miner; therefore, approval are obligatory. Crypto currency network arise in this classification where a miner can gain particular monetary incentives, for example Bitcoin, Ethereum, Lite coin, are crypto currency network established on community block-chain. In consortium sort of block-chain, simply nominated nodes are permitted to take part in dispersed consent procedure [43]. A few generous of productiveness can practice this sort of blockchain. Sometimes consortium block-chain are established for the specific trade (e.g. health-care segment), however exposed for community established on authorization. Private Block-chain are distributed system [43] where simply permission-able nodes can connect to the net. The undertaking of knobs as, to do trades, to implement nifty agreements, or to deed as mineworker, is organized in remote block-chain. Fundamentally, reliable group manages block-chain. Platform like Ripple [44], hyper ledger fabrics [45] merely provision private block-chain systems.

\subsection{Challenges of Blockchain in Healthcare}

Takes the lot is very challenging to integrate blockchain into health-care systems. Challenges Such as Administrator, the innovative issues need to be discussed. We have discussed several coherent challenges.

\subsubsection{Interoperability}

Sharing data easily and efficiently amongst different health care providers is a daunting job. On account of non-joint effort and absence of coordination [27], it turns into a hindrance to compelling information sharing. Patients and other medicinal services partners may confront issues with the information sharing and recuperation process. Overseeing and trading huge wellbeing records over human services offices isn't a simple undertaking while joining blockchain. As wellbeing information is of a delicate sort, it should just be imparted to confide in parties. An unapproved element must be guaranteed that it doesn't gain admittance to the information. To actualize blockchain in medicinal services, national enactment and information assurance should be clung to.

\subsubsection{Quality of Service (Qos)}

Another most significant issue concerning the implementation of blockchain is information must be given inside the fitting period. The lives of suffers could be at risk if the required data are not given inside the fitting time period. Since blockchain engineering is innately intricate, the consolidation of blockchain can cause computational postponement. Much work should be 
done to decrease that defer and better Qos proficiency before blockchain is incorporated into health care.

\subsubsection{Latency}

Latency is a crucial health-care parameter. Some technologies in healthcare are focused on making the diagnostic process faster on real-time monitoring. Before they are added, blocks are checked in the blockchain and this progression can cause a delay in getting to information and investigation than shared with different partners. Thus this delay must be considered when designing a health-care system based on blockchain.

\subsubsection{Constraints on resource and energy efficiency}

Blockchain adds complexities to calculations, cryptographic methodologies can be a weight on sensors [46]. Natural sensors are asset compelled gadgets regarding computational force, battery reinforcement, and so forth. So this high computational charge can cause an ascent in sensor temperature. This will trigger awkward awareness for the patient. Another problem is energy efficiency since the sensors are powered by batteries.

\subsubsection{Heterogeneous apparatuses and traffic}

Characteristic sensors are significant segments of medicinal services and these sensor gadgets produce different sorts of traffic. When all is said in done, information circulation is partitioned into two sorts' crisis traffic flow and customary traffic. Traffic delivered as of information collected from patients in a crisis circumstance is crisis traffic and, in routine observing, the information collected through devices is recognized as expected traffic. In this way, when incorporating blockchain in medicinal services, a need framework is a lot required, with the goal that the crisis traffic encounters an insignificant defer comparative with an ordinary vehicle.

\subsubsection{Scalability and Storage Capacity}

Since information generated by wellness sensors is colossal in quantity, such gigantic information should be stored by the blockchain's hubs competently. Well-being information can include clinical pictures, lab reports, Drug records, all of which require plenty of space for capacity. If distributed storage stages are used, this issue could be lightened.

\subsubsection{Data Mining}

Blockchain depends on the approval of information obstruct every information that originates from sensors is examined as a square of information and information delivered from the sensors every interval should be approved in advance connecting to a chain. In this way, the difficulty will emerge when the quantity of patients is expanded and all things. Effective mining is therefore also a very demanding problem as blockchain is incorporated into healthcare.

Another significant issue for consolidating blockchain in medicinal services is the unwavering quality of information assembled. Even though blockchain is well known because information put away in the blockchain is unchanging, once in a while information that originates from the sensors might be undermined so, the information will stay degenerate. Information got in the blockchain hubs might be modified, what's more, it may be conceivable given various security assaults like phony information infusion, listening stealthily, and so on. So care must be taken to ensure an effective security mechanism the data integrity.

\section{System Model}

A simple IoT-based medical care structure consists of three levels, the first level consisting of various medical devices such as pulse, blood pressure, ECG, etc. These devices are typically located on the patient's body. They are responsible for detecting various physical parameters from the patient's body, and then this data is directed to the PDA devices. In the second layer, PDA devices send information via an internet link to the medical server, and in the third layer doctors or medical facilities access the information. But the information in transmission is at risk for several cyber-attacks. Therefore, we require to implement authentication, privacy, access regulator, integrity. These four bounds are famous safety needs for the applications of medical care [47].

\subsection{Attack Model}

Attacks on confidentiality and integrity are two main types of attacks in traditional IoT-based applications. Confidentiality refers to the non-revealing of personal data of patients which is vulnerable to various dangers. Few general safety attacks on secrecy are imitation outbreak, Eavesdropping, packet sniffing, on the side network outbreak, etc. So, it's significant to control safety outbreaks in contradiction of privacy. Integrity confirms data retention during transmission. Today, IoT based biological instruments collect physical data from a patient's body, and that data is directed to health services then this information is transmitting over some insecure wireless/wired channels, it is easier for an enemy to remotely/materially capture the transmitting device and control the data collected using devices. As a consequence, it can tip to misdiagnosis. The most famous attacks on integrity are fake data injection attacks, replay attacks, data changing attacks, etc. In our recommended structure we have measured block-chain, which can protect outbreaks on reliability because of its working 
flora and many cryptographic techniques can be utilized to encrypt data at forwarder device to deal with attacks on confidentiality and the data can be decrypted by health service providers by using the secret key.

\section{Proposed Architecture}

At this point, we offer a smart and safe framework to effectively distribute the data by means of various medical amenities. The complete block-chain based design is presented in Fig. 4 where to store electronic medical record (EMR) cloud storage is used. In our recommended framework information collected by instruments is first forwarded to PDA device then the

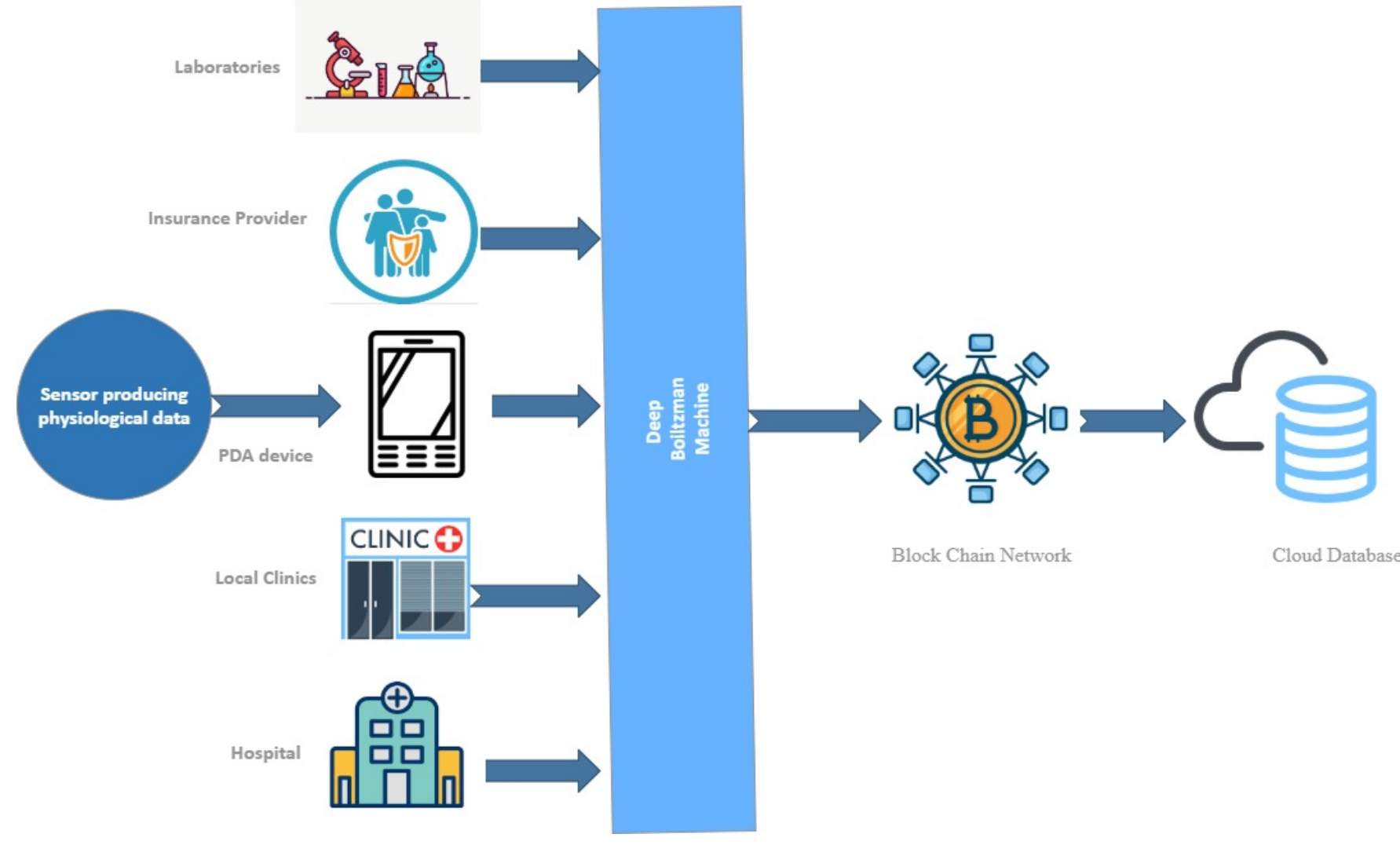

PDA device will produce the Hash of medical information by utilizing ordinary hash algorithms and later, via the internet the hash will be sent to a private block-chain link. Every health facility in block-chain such as labs, research labs, hospitals, insurance companies, etc. will entertain as block-chain node. PDA device directed Hash which will be accepted through every node and that block of data desires to be verified and authenticated using the nodes. Authentication is completed based on acknowledged Hash and that hash is matched by the hash of the recent data block usual. It's conceivable for the reason that the block of data produced through the PDA device may similarly hold the hash of the recent block. Most of the block-chain nodes required block authentication.

Figure 4. Proposed Block-chain established health data-sharing framework.

After verification, the block is added in the queue and an identifier (ID number), and a matchless clandestine key is produced. The ID and key are sent back to the PDA device. The actual medical data is encrypted by the PDA device by means of the key and the encoded data, ID, hash of the health-data are forwarded to the cloud-based database server. If anyone tries to damage the information of a single block, then the following blocks are correspondingly damaged. When a few health amenities want to get medical information stockpiled in the cloud, first the data is recognized by the ID and after this decoding is completed by means of the key. Just once decoding is completed, medical information becomes accessible. It's provided as a response in several Deep Learning-centered medical care application. We already talk about the different uses of Deep Learning systems for several medical care applications such as Medical Assessment Provision, Modified managements, Predictive healthcare, etc. The key benefit of the recommended design is the secure transmission of data between several health facilities and the information can be used in various applications related to health. In our design, safety demands are maintained. The data is accessed/stored by only authentic users when the block-chain is private. 
Cryptography algorithms support keeping the data confidential. Integrity is kept because of the block-chain working nature and the access-regulator is centered on a hidden key. As the hidden key is only produced through the nodes of the block-chain, so only they are allowed to decode data.

\section{Open Research Challenges}

In this Research work, we defined the use of block-chain and deep learning in medical. Block-chain and deep learning still have some open issues in the research area, appropriate studies can reduce this problem. It's already defined that different encryption-decryption algorithms such as AES, DES, 3 -DES are not a virtuous option to use in block-chain for medical care applications, by using these algorithms the delay in the relation of information distribution will increase. So simple cryptographic algorithm strategy is the main research zone. Key sharing and Key generation should be finished in a well-organized method so that it does not upsurge the difficulty of a block-chain based medical data-sharing framework. Furthermore, while designing a block-chain based safe medical care structure the number of investors may go on growing in IoT-supported remote medical care, report on high concerns, and storage on high concerns to be taken care. The information saved in EMR is mostly uncategorized, misplaced information, etc. Thus scholars should study the modernization of information from misplaced information, to remove noises data filtration is required. Moreover, medical data is very huge because of the large capacity of data. Growing scholars can discover making personal records based on their study ideas also using current standard databases considering concerned disorders, environmental position, demography, etc. of target issues to attain new accurate intelligent information handling outcomes. In this field there are too many research problems, complete research is required, to implement block-chain and deep learning techniques in medical data in a useful manner.

\section{Conclusion}

Now is the time of smart and remote applications in many sections, specifically in medical care, where several shareholders are associated with large medical records which need to be achieved, recovered, stored, in a scattered way via safety methods like block-chain, and managed logically by implementing deep learning methods. Challenges and problems remain in implementing deep learning methods as healthcare records are always incomplete, might be inaccurate, and not be characterized. Moreover, as medical data is large and multi-parties are associated, implementing all phases of block-chain techniques may lead toward communication overhead, extra storage overhead, and delays in processing data submission requests, therefore, making IoT supported real-time medical care help impractical. In this paper, we first described the basic concepts like Big data, IoT, Deep Learning, and blockchain to provide the understanding of the readers and then presented a novel solution to securely manage the hospital's big data using block-chain and deep learning. In the future, we will implement this system and evaluate it, and will present the results that how this proposed system is capable enough to manage hospital's big data securely.

\section{References}

1. Abdel-Zaher AM, Eldeib AM (2016) Breast cancer classification using deep belief networks. Expert Syst Appl 46:139-144. doi: 10.1016/j.eswa.2015.10.015

2. Andreu-Perez J, Poon CCY, Merrifield RD, Wong STC, Yang GZ (2015) Big Data for Health. IEEE J Biomed Heal Informatics 19:1193-1208. doi: 10.1109/JBHI.2015.2450362

3. Androulaki E, Barger A, Bortnikov V, Muralidharan S, Cachin C, Christidis K, De Caro A, Enyeart D, Murthy C, Ferris C, Laventman G, Manevich Y, Nguyen B, Sethi M, Singh G, Smith K, Sorniotti A, Stathakopoulou C, Vukolić M, Cocco SW, Yellick J (2018) Hyperledger Fabric: A Distributed Operating System for Permissioned Blockchains. Proc 13th EuroSys Conf EuroSys 2018 2018-Janua. doi: $10.1145 / 3190508.3190538$

4. Badža MM, Barjaktarović MC (2020) Classification of brain tumors from mri images using a convolutional neural network. Appl Sci 10. doi: 10.3390/app10061999

5. Bahga A, Madisetti VK (2016) Blockchain Platform for Industrial Internet of Things. J Softw Eng Appl 09:533-546. doi: 10.4236/jsea.2016.910036

6. Bar Y, Diamant I, Wolf L, Lieberman S, Konen E, Greenspan H (2018) Chest pathology identification using deep feature selection with non-medical training. Comput Methods Biomech Biomed Eng Imaging Vis 6:259-263. doi: 10.1080/21681163.2016.1138324

7. Barros PH, Lima BGC, Crispim FC, Vieira T, Missier P, Fonseca B (2018) Analyzing Social Network Images with Deep Learning Models to Fight Zika Virus. Lect Notes Comput Sci (including Subser Lect Notes Artif Intell Lect Notes Bioinformatics) 10882 LNCS:605610. doi: 10.1007/978-3-319-93000-8_69

8. Danaee P, Ghaeini R, Hendrix DA (2017) A deep 
learning approach for cancer detection and relevant gene identification. Pacific Symp Biocomput 0:219 229. doi: 10.1142/9789813207813_0022

9. Ekblaw, Ariel \& Azaria A (2017) MedRec: Medical Data Management on the Blockchain Our Motivation Approach From fragmented access to. 1-16

10. Esteva A, Kuprel B, Novoa RA, Ko J, Swetter SM, Blau HM, Thrun S (2017) Dermatologist-level classification of skin cancer with deep neural networks. Nature 542:115-118. doi: 10.1038/nature21056

11. Faust O, Hagiwara Y, Hong TJ, Lih OS, Acharya UR (2018) Deep learning for healthcare applications based on physiological signals: A review. Comput Methods Programs Biomed 161:1-13. doi: 10.1016/j.cmpb.2018.04.005

12. Fawaz HI, Forestier G, Weber J, Idoumghar L, Muller PA (2019) Adversarial Attacks on Deep Neural Networks for Time Series Classification. Proc Int Jt Conf Neural Networks 2019-July. doi: 10.1109/IJCNN.2019.8851936

13. Griggs KN, Ossipova O, Kohlios CP, Baccarini AN, Howson EA, Hayajneh T (2018) Healthcare Blockchain System Using Smart Contracts for Secure Automated Remote Patient Monitoring. J Med Syst 42:1-7. doi: 10.1007/s10916-018-0982-x

14. Jadhav S, Kasar R, Lade N, Patil M, Kolte S (2019) Disease Prediction by Machine Learning from Healthcare Communities. Int J Sci Res Sci Technol 2935. doi: $10.32628 /$ ijsrst19633

15. Karmakar K, Saif S, Biswas S, Neogy S (2018) WBAN Security: Study and implementation of a biological key based framework. Proc 5th Int Conf Emerg Appl Inf Technol EAIT 2018 1-6. doi: 10.1109/EAIT.2018.8470409

16. Kim BS, Kim KH, Kim K Il (2017) A survey on mobility support in wireless body area networks. Sensors (Switzerland) 17:1-18. doi: $10.3390 / \mathrm{s} 17040797$

17. Koga K, Spichkova M, Mantri N (2019) Towards automated management and analysis of heterogeneous data within cannabinoids domain. ENASE 2019 - Proc 14th Int Conf Eval Nov Approaches to Softw Eng 539546. doi: $10.5220 / 0007767405390546$

18. Kuo TT, Kim HE, Ohno-Machado L (2017) Blockchain distributed ledger technologies for biomedical and health care applications. J Am Med Informatics Assoc 24:1211-1220. doi: 10.1093/jamia/ocx068

19. Li R, Zhang W, Suk H Il, Wang L, Li J, Shen D, Ji S (2014) Deep learning based imaging data completion for improved brain disease diagnosis. Lect Notes Comput Sci (including Subser Lect Notes Artif Intell Lect Notes Bioinformatics) 8675 LNCS:305-312. doi: 10.1007/978-3-319-10443-0 39

20. Ma Y, Wang Y, Yang J, Miao Y, Li W (2017) Big Health Application System based on Health Internet of Things and Big Data. IEEE Access 5:7885-7897. doi: 10.1109/ACCESS.2016.2638449

21. Majumder S, Aghayi E, Noferesti M, MemarzadehTehran H, Mondal T, Pang Z, Deen MJ (2017) Smart homes for elderly healthcare-Recent advances and research challenges. Sensors (Switzerland) 17:32. doi $10.3390 / \mathrm{s} 17112496$

22. Mohsen H, El-Dahshan E-SA, El-Horbaty E-SM, Salem A-BM (2018) Classification using deep learning neural networks for brain tumors. Futur Comput Informatics J 3:68-71. doi: 10.1016/j.fcij.2017.12.001

23. Monti M, Rasmussen S (2017) RAIN: A Bio-Inspired Communication and Data Storage Infrastructure. Artif Life 23:552-557. doi: 10.1162/ARTL_a_00247

24. Mozaffari-Kermani M, Sur-Kolay S, Raghunathan A, Jha NK (2015) Systematic poisoning attacks on and defenses for machine learning in healthcare. IEEE J Biomed Heal Informatics 19:1893-1905. doi: 10.1109/JBHI.2014.2344095

25. Patel V (2019) A framework for secure and decentralized sharing of medical imaging data via blockchain consensus. Health Informatics J 25:13981411. doi: $10.1177 / 1460458218769699$

26. Ramsundar B, Kearnes S, Riley P, Webster D, Konerding D, Pande V (2015) Massively Multitask Networks for Drug Discovery

27. Ravi D, Wong C, Deligianni F, Berthelot M, AndreuPerez J, Lo B, Yang GZ (2017) Deep Learning for Health Informatics. IEEE J Biomed Heal Informatics 21:4-21. doi: 10.1109/JBHI.2016.2636665

28. Ronao CA, Cho SB (2016) Human activity recognition with smartphone sensors using deep learning neural networks. Expert Syst Appl 59:235-244. doi: 10.1016/j.eswa.2016.04.032

29. Shen B, Guo J, Yang Y (2019) MedChain: Efficient healthcare data sharing via blockchain. Appl Sci 9. doi: 10.3390/app9061207

30. Srinivas S, Sarvadevabhatla RK, Mopuri KR, Prabhu N, Kruthiventi SSS, Babu RV (2016) A taxonomy of deep convolutional neural nets for computer vision. Front Robot AI 2:1-13. doi: 10.3389/frobt.2015.00036

31. Sun W, Zheng B, Qian W (2016) Computer aided lung 
cancer diagnosis with deep learning algorithms. Med Imaging 2016 Comput Diagnosis 9785:97850Z. doi: $10.1117 / 12.2216307$

32. Tsai CW, Lai CF, Chiang MC, Yang LT (2014) Data mining for internet of things: A survey. IEEE Commun Surv Tutorials 16:77-97. doi: 10.1109/SURV.2013.103013.00206

33. Xia Q, Sifah EB, Asamoah KO, Gao J, Du X, Guizani M (2017) MeDShare: Trust-Less Medical Data Sharing among Cloud Service Providers via Blockchain. IEEE Access 5:14757-14767. doi: 10.1109/ACCESS.2017.2730843

34. Yin XC, Liu ZG, Ndibanje B, Nkenyereye L, Islam SMR (2019) An iot-based anonymous function for security and privacy in healthcare sensor networks. Sensors (Switzerland) 19:1-14. doi: $10.3390 / \mathrm{s} 19143146$

35. Zhang P, White J, Schmidt DC, Lenz G, Rosenbloom ST (2018) FHIRChain: Applying Blockchain to Securely and Scalably Share Clinical Data. Comput Struct Biotechnol J 16:267-278. doi: 10.1016/j.csbj.2018.07.004

36. Zheng Z, Xie S, Dai H, Chen X, Wang H (2017) An Overview of Blockchain Technology: Architecture, Consensus, and Future Trends. Proc - 2017 IEEE 6th Int Congr Big Data, BigData Congr 2017 557-564. doi: 10.1109/BigDataCongress.2017.85

37. Artelnics: Neural designer (2015). Available online: https://www.neuraldesigner.com
38. . Chollet, F.: Keras (2016). Available online: https://keras.io/

39. . Apache Software Foundation: Apache Singa (2016). Available online: https://singa.incubator. apache.org

40. Skymind: Deeplearning4j (2016). Available online: http://deeplearning4j.org

41. Microsoft: Microsoft cognitive toolkit (2016). Available Online: https://github.com/microsoft/ cntk

42. Apache Software Foundation: Apache MXNet (2016). Available Online: https://mxnet.apache. org/

43. Artelnics: OpenNN (2014). Avaiable Online: http://www.opennn.net

44. Paszke, A, Gross, S., Chintala, S., Chanan, G.: PyTorch (2016). Avaiable Online: https:// pytorch.org

45. Google: Tensorflow (2016). Available Online: https://www.tensorflow.org

46. Universite de Montreal: Theano (2019). Available Online: http://deeplearning.net/software/ theano/

47. Saif, S., Gupta, R., Biswas, S.: Implementation of cloud assisted secure data transmission in WBAN for healthcare monitoring. In: Proceedings of International Conference on Advanced Computational and Communication Paradigms (ICACCP 2017), Advances in Inte 